\title{
Rapid Design, Fabrication, and Optimization of 3D Printed Photonic Topological Insulators
}

Michael S. Mattei ${ }^{1}$, Boyuan Liu' ${ }^{2}$, Gerardo A. Mazzei Capote ${ }^{3}$, Zijie Liu ${ }^{3}$, Tim A. Osswald , Zongfu $\mathrm{Yu}^{2}$, Randall H. Goldsmith*1

M. S. Mattei, R. H. Goldsmith

Department of Chemistry, University of Wisconsin-Madison

1101 University Avenue, Madison, WI, 53706, USA

E-mail: rhg@chem.wisc.edu

B. Liu, Z. Yu

Department of Electrical and Computer Engineering, University of Wisconsin-Madison

1415 Engineering Drive, Madison, WI, 53703, USA

G. A. Mazzei Capote, Z. Liu, T. A. Osswald

Department of Mechanical Engineering, University of Wisconsin-Madison

1513 University Avenue, Madison, WI, 53703, USA

Keywords: Topological Photonics, Additive Manufacturing, High-Speed FDTD

Abstract: Photonic topological insulators have emerged as an exciting new platform for backscatter-free waveguiding even in the presence of defects, with applications in robust longrange energy and quantum information transfer, low-threshold lasing, and chiral quantum optics. We demonstrate a design for spin-Hall photonic topological insulators with remarkably low refractive index contrast, enabling the synthesis of photonic topological waveguides from polymeric materials for the first time. Our design is compatible with additive manufacturing methods, including fused filament fabrication, and constitutes the first demonstration of a 3D printed photonic topological insulator. We combine rapid device fabrication through 3D printing with high-speed FDTD simulation to showcase an iterative design cycle capable of screening thousands of device configurations with unprecedented speed. We have identified and experimentally verified a non-intuitive geometry for bent topological waveguides with optimized transmission. Our rapid design cycle represents a powerful new tool set for designing, optimizing, and synthesizing photonic topological materials.

\section{Introduction}

Photonic topological insulators (TIs), periodic structures with a band gap in the bulk but gapless dispersion at the edges, possess the unique ability to guide light around defects and sharp turns without scattering. ${ }^{[1-3]}$ Such topological protection of light propagation provides an exciting new platform for on-chip low-threshold lasing, ${ }^{[4-6]}$ robust long-range quantum information and energy transfer, ${ }^{[7-9]}$ unidirectional emission from spin-polarized quantum states, ${ }^{[10]}$ robust optical resonators and delay lines, ${ }^{[11,12]}$ soliton generation, ${ }^{[13,14]}$ and quantum networks. ${ }^{[7,15,16]}$ Photonic TIs were first demonstrated at microwave frequencies by breaking time-reversal symmetry in a lattice of gyromagnetic rods, producing the photonic analogue of the quantum Hall effect ${ }^{[17]}$ which produces a single unidirectional chiral edge state. The absence of a counter-propagating mode ensures that the edge state is robust against backscattering defects. Achieving all-dielectric 
designs for photonic TIs has required mimicking the spin-Hall, ${ }^{[18]}$ and valley-Hall effects. ${ }^{[12]}$ In such systems, two counterpropagating helical edge states exist which are decoupled via their opposite spin and valley angular momenta, respectively. Thus, the helical edge states in spin and valley Hall systems are only robust against perturbations which preserve the spin degree of freedom. ${ }^{[11]}$ Indeed, in the only experimental example of quantifying topological protection in dielectric bent topological waveguides, there is still a small amount of measurable loss due to reflection from the corners. ${ }^{[19]}$

As topological photonic technologies mature, it is important to be able to rapidly test and quantify performance, both via simulation and experiment, so that best practices in design can be established. Due to the self-similarity of photonic structures, with many properties only relying on the ratio of wavelength to unit cell, this challenge spans a tremendous diversity of materials systems and energy scales. To begin to address this need, we present the rapid design, simulation, and fabrication of polymeric photonic TIs at microwave frequencies using an iterative cycle of high-speed finite-difference time-domain simulation (FDTD), fused filament fabrication (FFF), and microwave transmission measurements to optimize transmission through omega-shaped topological waveguides. ${ }^{[2,11,19]}$ In the process, we present a photonic topological insulator design that requires substantially lower refractive index contrast, thus enabling access to the many advantages of 3D printing in polymeric materials, and showcase a new optical simulation approach with vastly improved speed. With these innovations, we report the first photonic topological insulator fabricated from polymeric materials, and the first 3D printed photonic topological insulator. Taken together, these approaches add a powerful new set of tools for the design, optimization, and synthesis topological photonic materials.

As an example of our iterative design and synthesis approach, we examine the nature of the corners of omega-shaped bent topological waveguides. For all-dielectric topological systems without time reversal symmetry breaking due to magnetoptical effects, it is important to quantify the impact of sharp turns and defects on the transmission of waveguides and resonators in order to optimize device performance. ${ }^{[20]}$ We screened 4096 possible corner geometries for such waveguides, a process that would have been prohibitively slow with typical simulation software, and found a spread in waveguide transmission of more than $73 \%$. We have identified a nonintuitive optimized bent waveguide geometry and experimentally verified its high transmission compared to poorly transmitting geometries. Our approach thus reveals the power of high-speed simulation and additive manufacturing in quantifying topological protection and optimizing topological structures.

\section{Results and Discussion}

We first present a design for two-dimensional (2D) spin-Hall photonic TIs with remarkably low refractive index contrast (as low as 1.55), greatly expanding the materials pallet available for fabricating photonic TIs, including polymers. Polymeric materials provide a number of advantages as media for topological photonics including (often) low absorption at visible wavelengths, straightforward doping of quantum emitters and gain materials into the topological lattice, application in flexible photonic devices, and especially compatibility with rapid fabrication techniques such as additive manufacturing and soft lithography. Our design (Figure 1a) is an adaptation of the expanded honeycomb lattice of rods, ${ }^{[18]}$ with the rod dimensions and degree of expansion carefully tuned to maintain a complete topological band gap with low index contrast. At a ratio $\mathrm{a}_{0} / \mathrm{R}$ equal to 3 the honeycomb lattice supports a Dirac point between bands 2 and 3. Decreasing the $\mathrm{a}_{0} / \mathrm{R}$ opens the Dirac point into a topological band gap, while increasing 
the ratio yields a trivial bandgap. We found complete photonic band gaps for structures with refractive index as low as $n=1.55$ with $\mathrm{a}_{0} / \mathrm{R}=2.4$ for the topological lattice (Figure $1 \mathrm{a}, \mathrm{b}$ ) and $\mathrm{a}_{0} / \mathrm{R}=4.4$ for its trivial counterpart (Figure S1). In contrast, previously demonstrated photonic topological insulators have been fabricated from materials with much higher index, such as silicon nitride in the visible ${ }^{[21]}(n=2.0)$ and alumina at microwave frequencies $(n=3.1){ }^{[22]}$ Our design thus greatly expands the pallet of available materials for producing photonic topological insulators, including polymers at optical, infrared, and microwave frequencies, and oxides at optical frequencies.
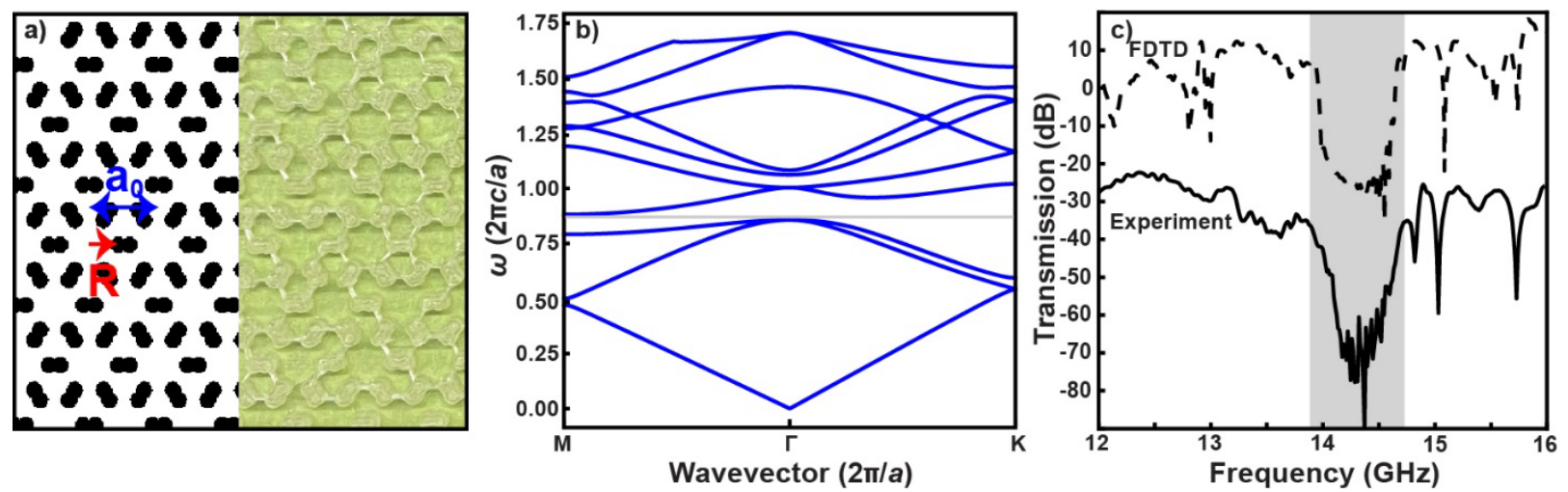

Figure 1. (a) Structure of the expanded honeycomb topological insulator explored in this work, with the 3D printed structure overlaid on the right. (b) Band structure of an infinite honeycomb TI lattice. The complete topological band gap is highlighted in grey. (c) Theoretical and experimental transmission spectra of a finite (24x15 unit cells) expanded honeycomb structure along the $\mathrm{M}$ direction, with the band gap region highlighted in grey.

Using FFF, we fabricated the expanded structure $\left(\mathrm{a}_{0}=17.8 \mathrm{~mm}, 7.35 \mathrm{~mm}\right.$ height, Figure 1a overlay) from polylactic acid (PLA) on an aluminum substrate. FFF provides a rapid, automated and highly reproducible means of fabricating complex geometries from thermoplastic polymeric materials, allowing straightforward introduction of arbitrary defects into the structure. FFF also provides a tradeoff between print time and part density (which affects refractive index) by tuning the infill percentage. For PLA (bulk $n=1.64$ ), maintaining a refractive index above 1.55 for reasonable print times was a key technical challenge in this work. We have selected slicing parameters (Table S1) which result in print times of $\sim 30$ largely unsupervised hours while achieving a refractive index of $\sim 1.61$, as determined from comparison to the simulated transmission spectrum. Specialty filaments with high refractive index ${ }^{[23,24]}$ could be used to produce structures with lower infill percentage and thus lower density, further reducing print times and providing a flexible means for introducing refractive index gradients as a degree of freedom in device design.

We then measured the transmission along $\mathrm{M}$ using a parallel plate waveguide apparatus and observed a $\sim 40 \mathrm{~dB}$ dip in the spectrum at $\sim 14.35 \mathrm{GHz}$ corresponding to the topological band gap, in agreement with high-speed FDTD simulation (Figure 1c). The interface between the topological and trivial structures supports a topological edge state which bridges the band gap. The "armchair" interface between our topological and trivial structures is depicted in Figure 2a with the electric field $\left(E_{z}\right)$ distribution of the topological edge state overlaid, and the corresponding band structure is shown in Figure $2 \mathrm{~b}$ with the edge states shown in red. These edge states are topologically protected against backscattering from defects which preserve the angular momentum of the wave, and should thus navigate sharp turns in the interface. Figure 2c shows a bent "omega" waveguide, with the corresponding topological edge states navigating a Z- 
shaped turn without loss. Indeed, experimental transmission measurements show high transmission across the entire band gap region, with comparable transmission for the straight and bent waveguides, indicative of topological protection and excellent agreement with high-speed FDTD simulations (Figure 2d). This constitutes the first experimental example of a polymeric photonic topological insulator, greatly expanding the range of available materials and fabrication methods for designing photonic topological devices.
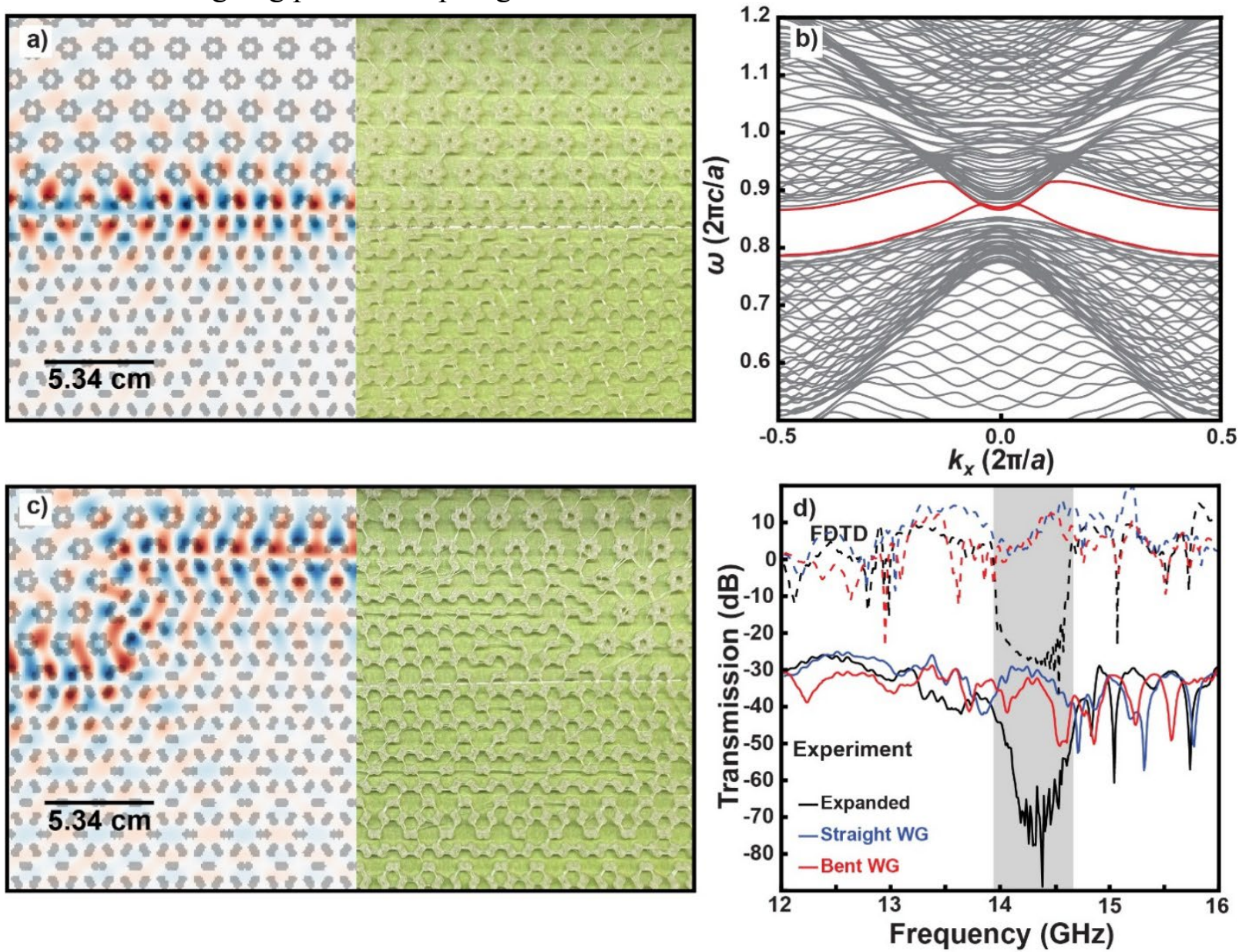

Figure 2. (a) The straight interface between the topological and trivial domains hosts a topological edge state. The $E_{Z}$ component of the edge state shows that the mode is well confined to the straight waveguide. The 3D printed structure is overlaid on the right side. (b) Calculated supercell band structure for the straight waveguide in (a), depicting the gapless topological edge states in red. (c) The topological edge states propagate around sharp turns in a bent "omega" waveguide without backscattering. (d) Simulated and experimental transmission spectra showing high transmission through both the straight and bent topological waveguides in the band gap region (highlighted in grey).

Similar demonstrations of topologically protected propagation around sharp turns have been published previously. ${ }^{[2,11,17,19]}$ However, there has been no systematic investigation of the influence of corner geometry on the transmission through bent topological waveguides. Such an investigation, in order to find the global maximum, would require a prohibitively large number of simulations and syntheses. Our bent waveguide has two unique pairs of corners, each of which is a combination of the topological and trivial unit cell. Thus, there are $2^{12}=4096$ possible corner configurations. We simulated the transmission through all 4096 possible structures using a highspeed FDTD solver (Tidy3D) with a total simulation time of 14.8 hours (13 seconds per simulation). We timed a 2D simulation using a commercial FDTD software with the parameters 
set as closely as possible to those used in our Tidy3D simulations and measured a simulation time of 5.81 minutes. Running 4096 such simulations would thus require 2.4 weeks of simulation time (a $24 \mathrm{x}$ increase). A summary of the optimization is shown in Figure 3.

The bent waveguide design in Figure 2 has corner configurations similar to those used in previous works. ${ }^{[25,26]}$ This structure is the most intuitive, as each unit cell of one constituent lattice along the interface is complete. In contrast, we find that the structure with optimized transmission deviates from this intuitive design. The structure of this optimized waveguide is shown in Figure 3a (Structure (i), left panel), in which one of the rods from the topological lattice has instead been placed in its corresponding location in the trivial lattice, and neither the topological nor the trivial unit cell is complete at the corner. This clearly demonstrates that rapid simulation can be a powerful design tool for optimizing the performance of topological photonic crystals, where the optimal device geometry is not necessarily intuitive. In contrast, we also present a device configuration with comparatively low transmission. The defects present in Structure (ii) and (iii) (Figure 3a, center and right panels) do not preserve the spin degree of freedom, and thus induce backscattering in the waveguide. Comparison (Figure 3b) between the calculated transmission spectra and the experimentally measured spectra in 3D printed samples confirms that Structures (ii) and (iii) indeed contain defects against which the waveguide mode is not topologically protected.

Figure 3c shows a histogram of the transmission through all 4096 simulated topological waveguides relative to the optimized structure. The distribution is continuous and appears to be multimodal. The multimodal nature of the distribution likely arises from the fact that we have only considered two discrete possible positions for each rod, and thus a discrete number of possible transmission values for each corner. More striking is the large spread in transmission values (a range of $73 \%$ ), which suggests that a majority of the simulated geometries induce significant backscattering, and thus do not preserve the spin angular momentum of the wave. However, because the distribution is continuous, it is unclear where the cutoff between spinpreserving and spin-flipping defects resides, and thus whether high transmission through a bent waveguide is sufficient for verifying topological protection. Some features which are common in low transmission geometries and rare in high transmission configurations are shown in Figure S2. However, no intuitive design rules are apparent from examining the structures of the worst performing waveguides compared to those with the highest transmission. Indeed, the structure in Figure S2 possessing all three of the identified detrimental features has a relative transmission of $-35.9 \%$ compared to the optimized structure, while the structure with only one of the features has a relative transmission of $-47.3 \%$, demonstrating that while there are some qualitative similarities between low-transmission designs, there is no obvious quantitative trend. To circumvent this challenge, we propose that our rapid screening method combined with rapid experimental validation via additive manufacturing can be used to generate training sets for machine learning algorithms which can further optimize the performance of topological photonic devices by leveraging nonintuitive correlations between structure and transmission. 

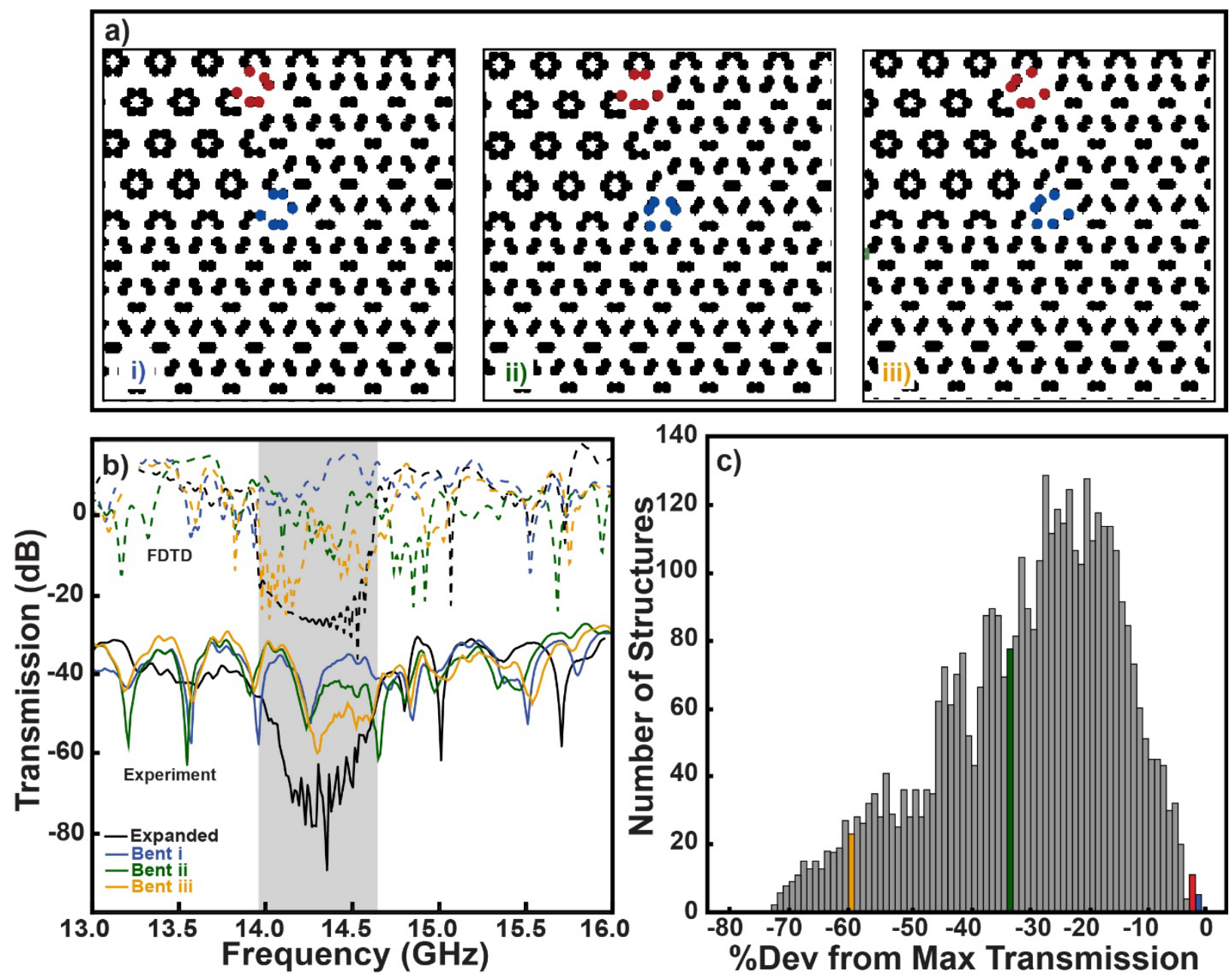

Figure 3. (a) Examples of 3 out of 4096 possible corner configurations for the bent topological waveguide. Structure (i) has the highest total transmission across the band gap. Structure (ii) is an example of a defect which does not preserve the angular momentum degree of freedom and thus has lower transmission. Structure (iii) has still lower transmission. (b) Simulated and experimental transmission spectra for the 3 structures in (a). The experimental spectra verify that Structures (ii) and (3) indeed have lower transmission compared to Structure (i). The band gap of the expanded structure is shown for reference. (c) Histogram of the percent deviation (linear scale, from simulation) for the total transmission through all 4096 possible structures relative to the structure with the highest total transmission. The position of each structure is color coded in the histogram. The red bar indicates the transmission of the original bent waveguide from Figure 2.

\section{Conclusions}

We have developed a design for polymeric photonic topological insulators with remarkably low refractive index (as low as1.55). Our design is compatible with and realized by additive manufacturing techniques, allowing rapid prototyping of photonic devices with flexible design. We combined this rapid fabrication capability with high-speed FDTD simulation to screen 4096 possible corner configurations for a bent topological waveguide. Our high-throughput design cycle revealed a nonintuitive structure optimized for the highest transmission. High-speed simulation combined with additive manufacturing can provide an ideal platform for exploring and optimizing novel designs for topological photonics, and generating training sets for designing topological photonic devices via machine learning. 


\section{Experimental Section}

\subsection{Band Structure and Transmission Calculations}

Band structures of the 2D topological and trivial band gap structures were performed using the MIT Photonic Bandgap (MPB) Python package, treating both structures as an infinite lattice. ${ }^{[27]}$ The band structure for the straight waveguide was also calculated using MPB, treating the structure as an infinite lattice along the waveguide (X) axis and a finite supercell (10 topological unit cells and 10 trivial unit cells) along the $\mathrm{Y}$ axis.

In-plane transmission simulations were performed using Tidy3D (Flexcompute, Inc.) with a point source polarized along $\mathrm{Z}$ (perpendicular to the plane of the crystal). The $\mathrm{X}$ and $\mathrm{Y}$ boundary conditions were set to the built-in 'absorber' option, and the $Z$ boundary condition was periodic. A perfect electrical conductor (PEC) layer was placed above and below the crystal to approximate the parallel plate waveguide. The high-accuracy simulations compared with our experimental measurements were performed using 128 mesh cells per unit cell along $\mathrm{X}$ and $\mathrm{Y}$, and 3 mesh cells along Z (one mesh cell for each PEC layer and one for the crystal, effectively a 2D simulation). The 4096 high-speed simulations for optimizing corner geometry were performed using 32 mesh cells per unit cell along $\mathrm{X}$ and $\mathrm{Y}$, and one mesh cell each for the crystal and PEC layers along $Z$. The bent waveguide structure possesses mirror symmetry across the plane perpendicular to the propagation axis. For the corner optimization process, we therefore simulated only the first half of the bent waveguide to decrease simulation time. All transmission spectra were acquired by measuring the electric field amplitude at a time-domain monitor and taking the Fourier transform and normalizing by subtracting the simulated spectrum with no crystal present.

\subsection{Additive Manufacturing of Microwave Topological Insulators}

An FFF system (FusedForm FF600) was used to 3D print our topological insulator samples from polylactic acid (Matterhackers "Natural PLA PRO", $1.75 \mathrm{~mm}$ diameter) using a $0.8 \mathrm{~mm}$ nozzle and a layer height of $0.2 \mathrm{~mm}$. Care was taken to ensure that all the acquired spools of filament came from the same lot to avoid variations in material properties from sample to sample. All samples were printed on 1/16 inch thick aluminum substrates, which do not influence the transmission through the sample. Green painter's tape (3M Scotch 401+ Masking Tape) was applied to the aluminum sheet prior to printing to improve adhesion of the PLA to the substrate. Toolpath generation was performed using the Simplify3D slicing engine (v4.1.2). The slicing parameters (Table S1) were selected iteratively to maximize the refractive index of the printed part while maintaining fidelity of the geometric features and constraining print times to $\sim 33$ hours. Common defects that arise due to the printing process included polymer drool between adjacent structures, unwanted z-height deposition that resulted in inconsistent height of the structure, voids in the areas where contiguous circular beads meet, as well as warpage. These issues were mitigated through manipulation of the slicing engine parameters, resulting in the structures used in this work.

\subsection{Transmission Measurements of Microwave Topological Insulators}

In-plane transmission measurements of microwave TIs were performed in a parallel plate waveguide using a Keysight E5071C network analyzer with antennas from Fairview Microwave (SKU: SC3792). The sample is surrounded by radiofrequency absorbing foam and placed 
between two aluminum plates. The antennas are fixed to the top aluminum plate and spaced 35.8 $\mathrm{cm}$ apart along the long axis of the waveguide so that both antennas reside inside the photonic crystal (Figure S3).

\section{Acknowledgments}

This research was supported by the National Science Foundation through the University of Wisconsin Materials Research Science and Engineering Center (DMR-1720415). The authors thank Professor Nader Behdad and Professor Yin Poo for helpful discussions of the parallel plate waveguide transmission measurements.

\section{Conflict of Interest}

The authors declare no conflict of interest.

\section{References}


[1] L. Lu, J. D. Joannopoulos, M. Soljačić, Nat. Photonics 2014, 8, 821.

[2] A. B. Khanikaev, G. Shvets, Nat. Photonics 2017, 11, 763.

[3] T. Ozawa, H. M. Price, A. Amo, N. Goldman, M. Hafezi, L. Lu, M. C. Rechtsman, D. Schuster, J. Simon, O. Zilberberg, I. Carusotto, Rev. Mod. Phys. 2019, 91, 015006.

[4] M. A. Bandres, S. Wittek, G. Harari, M. Parto, J. Ren, M. Segev, D. N. Christodoulides, M. Khajavikhan, Science. 2018, 359, DOI 10.1126/science.aar4005.

[5] L. Pilozzi, C. Conti, Phys. Rev. B 2016, 93, 195317.

[6] Y. Gong, S. Wong, A. J. Bennett, D. L. Huffaker, S. S. Oh, ACS Photonics 2020, 7, 2089.

[7] S. A. H. Gangaraj, G. W. Hanson, M. Antezza, Phys. Rev. A 2017, 95, 1.

[8] P. Doyeux, S. A. H. Gangaraj, G. W. Hanson, M. Antezza, Phys. Rev. Lett. 2017, 119, 2.

[9] Y. Wang, J. Ren, W. Zhang, L. He, X. Zhang, Phys. Rev. Appl. 2020, 14, 054007.

[10] J.-W. Dong, X.-D. Chen, H. Zhu, Y. Wang, X. Zhang, Nat. Mater. 2017163 2016, 16, 298.

[11] K. Lai, T. Ma, X. Bo, S. Anlage, G. Shvets, Sci. Rep. 2016, 6, 1.

[12] T. Ma, G. Shvets, New J. Phys. 2016, 18, 025012.

[13] D. Leykam, Y. D. Chong, Phys. Rev. Lett. 2016, 117, 1.

[14] S. Mukherjee, M. C. Rechtsman, Science. 2020, 368, 856.

[15] M. C. Rechtsman, Y. Lumer, Y. Plotnik, A. Perez-Leija, A. Szameit, M. Segev, Conf. Lasers Electro-Optics Eur. - Tech. Dig. 2015, 2015-Augus.

[16] P. Lodahl, S. Mahmoodian, S. Stobbe, A. Rauschenbeutel, P. Schneeweiss, J. Volz, H. Pichler, P. Zoller, Nat. 20175417638 2017, 541, 473.

[17] Z. Wang, Y. D. Chong, J. D. Joannopoulos, M. Soljačić, Phys. Rev. Lett. 2008, 100, 013905.

[18] L.-H. Wu, X. Hu, 2015, DOI 10.1103/PhysRevLett.114.223901.

[19] S. Arora, T. Bauer, R. Barczyk, E. Verhagen, L. Kuipers, Light Sci. Appl. 2021101 2021, 10,1 .

[20] R. E. Christiansen, F. Wang, O. Sigmund, S. Stobbe, Nanophotonics 2019, 8, 1363.

[21] W. Liu, M. Hwang, Z. Ji, Y. Wang, G. Modi, R. Agarwal, Nano Lett. 2020, 20, 1329.

[22] X. D. Chen, W. M. Deng, F. L. Shi, F. L. Zhao, M. Chen, J. W. Dong, Phys. Rev. Lett. 2019, 122, 233902.

[23] Y. Wu, D. Isakov, P. S. Grant, Materials (Basel). 2017, 10, DOI 10.3390/ma10101218.

[24] F. Castles, D. Isakov, A. Lui, Q. Lei, C. E. J. Dancer, Y. Wang, J. M. Janurudin, S. C. Speller, C. R. M. Grovenor, P. S. Grant, Sci. Reports 201661 2016, 6, 1.

[25] B. Xie, G. Su, H.-F. Wang, F. Liu, L. Hu, S.-Y. Yu, P. Zhan, M.-H. Lu, Z. Wang, Y.-F. Chen, Nat. Commun. 2020111 2020, 11, 1.

[26] Y. Peng, B. Yan, J. Xie, E. Liu, H. Li, R. Ge, F. Gao, J. Liu, Phys. status solidi-Rapid Res. Lett. 2020, 14, 2000202.

[27] J. D. Joannopoulos, S. G. Johnson, Opt. Express, Vol. 8, Issue 3, pp. 173-190 2001, 8, 173. 